\title{
Three-dimensional pelvic incidence is much higher in (thoraco)lumbar scoliosis than in controls
}

\author{
Rob C. Brink ${ }^{1}$ (D - Ludvig Vavruch ${ }^{2,3} \cdot$ Tom P. C. Schlösser $^{1} \cdot$ Kasim Abul-Kasim $^{4} \cdot$ Acke Ohlin $^{5} \cdot$ Hans Tropp $^{2,3}$. \\ René M. Castelein ${ }^{1} \cdot$ Tomaž Vrtovec $^{6}$
}

Received: 13 October 2017 / Revised: 25 June 2018 / Accepted: 4 August 2018 / Published online: 20 August 2018

(c) The Author(s) 2018

\begin{abstract}
Purpose The pelvic incidence (PI) is used to describe the sagittal spino-pelvic alignment. In previous studies, radiographs were used, leading to less accuracy in establishing the three-dimensional (3D) spino-pelvic parameters. The purpose of this study is to analyze the differences in the 3D sagittal spino-pelvic alignment in adolescent idiopathic scoliosis (AIS) subjects and non-scoliotic controls.

Methods Thirty-seven female AIS patients that underwent preoperative supine low-dose computed tomography imaging of the spine, hips and pelvis as part of their general workup were included and compared to 44 non-scoliotic age-matched female controls. A previously validated computerized method was used to measure the PI in 3D, as the angle between the line orthogonal to the inclination of the sacral endplate and the line connecting the center of the sacral endplate with the hip axis. Results The PI was on average $46.8^{\circ} \pm 12.4^{\circ}$ in AIS patients and $41.3^{\circ} \pm 11.4^{\circ}$ in controls $(p=0.025)$, with a higher PI in Lenke type 5 curves $\left(50.6^{\circ} \pm 16.2^{\circ}\right)$ as compared to controls $(p=0.042)$, whereas the Lenke type 1 curves $\left(45.9^{\circ} \pm 12.2^{\circ}\right)$ did not differ from controls $(p=0.141)$.

Conclusion Lenke type 5 curves show a significantly higher PI than controls, whereas the Lenke type 1 curves did not differ from controls. This suggests a role of pelvic morphology and spino-pelvic alignment in the pathogenesis of idiopathic scoliosis. Further longitudinal studies should explore the exact role of the PI in the initiation and progression of different AIS types.
\end{abstract}

Graphical abstract These slides can be retrieved under Electronic Supplementary Material.
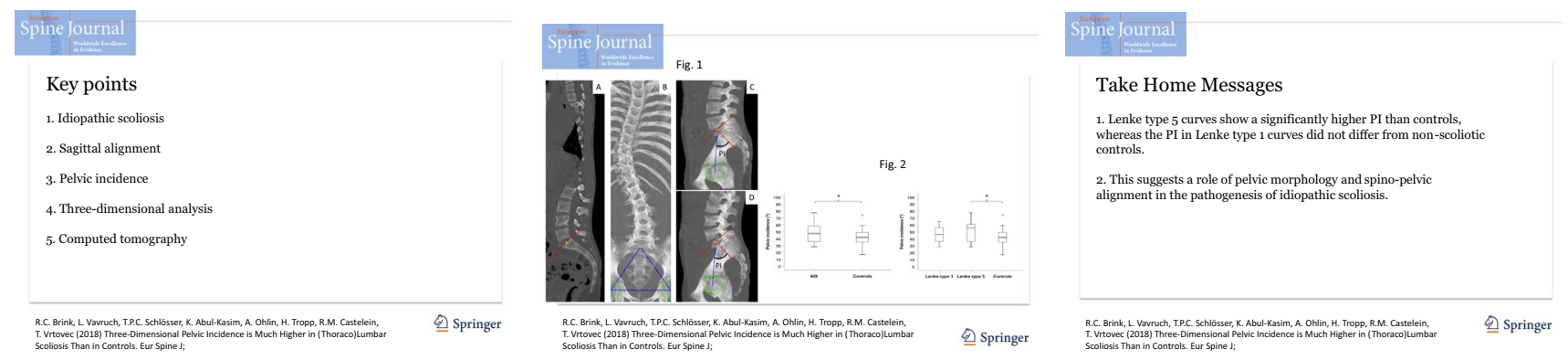

Keywords Idiopathic scoliosis $\cdot$ Sagittal alignment $\cdot$ Pelvic incidence $\cdot$ Three-dimensional analysis $\cdot$ Computed tomography

Rob C. Brink and Ludvig Vavruch contributed equally and are co-first authors.

Electronic supplementary material The online version of this article (https://doi.org/10.1007/s00586-018-5718-6) contains supplementary material, which is available to authorized users.

Extended author information available on the last page of the article

\section{Introduction}

Upright human spinal biomechanics and the sagittal shape of the spine play an important role in the development and progression of spinal deformities such as adolescent idiopathic scoliosis (AIS) [1-7]. In 1985, During et al. 
[8] described the pelvic sacral angle, and later on, DuvalBeaupère et al. [9] used the term pelvic incidence (PI) for a parameter that defines the relationship between the pelvic anatomy and spinal alignment. Several other authors described the spino-pelvic balance in normal children and adolescents during growth and in patients with different pathologies [1, 10-21]. Dickson et al. [5] described that differences in the sagittal plane (reversal of the normal thoracic kyphosis) during growth could initiate a progressive idiopathic scoliosis. Moreover, previous authors suggested a link between the spino-pelvic morphology and spinal deformities in the sagittal plane [1, 10-21]. Most of the previous studies obtained two-dimensional (2D) referential values using standing lateral radiographs that could lead to a deviation from the true sagittal plane and to a certain degree of distortion of the image. Three-dimensional (3D) images are not affected by the characteristics of radiographic imaging, like the projection plane, and can thus better observe the relationship between the sacrum, pelvis and hips [22]. Pasha et al. [14] introduced novel pelvic parameters based on 3D reconstructed radiographs, and Vrtovec et al. [22] measured the 3D PI in a non-scoliotic population using computed tomography (CT) scans, demonstrating the improved accuracy of this method as compared to the traditional measurements on plain sagittal $\mathrm{X}$-rays. In previous studies, the exact difference in pelvic morphology between AIS subjects and non-scoliotic controls has remained unclear. Therefore, the purpose of this study was to analyze, using a previously validated accurate 3D technique, the differences in sagittal pelvic morphology between AIS patients and asymptomatic adolescents.

\section{Materials and methods}

\section{Study population}

This study, the purpose of which is to analyze the differences in 3D sagittal pelvic morphology between AIS patients and non-scoliotic controls, has been approved by the local research ethics committee. Female patients with thoracic right convex and (thoraco)lumbar left convex AIS requiring surgery between August 2009 and May 2018 were included (demographics in Table 1). All had undergone preoperative supine low-dose (20 times lower than that of a standard CT scan) CT imaging of the spine at two institutions (Linkoping and Malmö, Sweden) [23, 24]. The low-dose CT scan is part of the standard preoperative protocol in both centers. Patients were included if, in addition to the spine, both femoral heads were visible on the CT scan. Subjects with other spinal pathologies, a left convex thoracic/right convex (thoraco)lumbar curve and/or previous spinal surgery were excluded. Curve characteristics, including the Cobb angle, thoracic kyphosis (TK: T4-T12) and lumbar lordosis (LL: T12-S1), were determined on the conventional posterior-anterior and lateral radiographs as well as on digitally reconstructed lateral radiographs (DRRs) of the supine CT scans (Table 1). To represent the normal population, a second group was included that consisted of non-scoliotic agematched female subjects that were selected from a preexisting database who had undergone CT imaging of the thorax and abdomen for indications other than spinal pathology (for example, trauma screening). The non-scoliotic observed cohort was assumed to represent a normal population, since a number of subjects were already excluded from the
Table 1 Demographics for all included adolescent idiopathic scoliotic (AIS) patients and controls

\begin{tabular}{llll}
\hline & & AIS patients $(n=37)$ & Controls $(n=44)$ \\
\hline Age & Range & $12-21$ years & $12-21$ years \\
& Mean \pm SD & $15.4 \pm 2.4$ years & $15.4 \pm 2.9$ years \\
Females & Number (ratio) & $37(100 \%)$ & $44(100 \%)$ \\
Thoracic curve right convexity & Number (ratio) & $37(100 \%)$ & - \\
Thoracic Cobb angle & Range & $21-84^{\circ}$ & - \\
& Mean \pm SD & $50.3 \pm 14.6^{\circ}$ & - \\
(Thoraco)lumbar Cobb angle & Range & $19-78^{\circ}$ & - \\
& Mean \pm SD & $46.5 \pm 13.8^{\circ}$ & - \\
Lenke curve & & & - \\
Type 1 & Number (ratio) & $21(57 \%)$ & - \\
Type 2 & Number (ratio) & $1(3 \%)$ & - \\
Type 3 & Number (ratio) & $2(5 \%)$ & - \\
Type 5 & Number (ratio) & $9(24 \%)$ & - \\
Type 6 & Number (ratio) & $4(11 \%)$ & - \\
\hline
\end{tabular}

Curve characteristics, measured on upright lateral radiographs, are also shown for AIS patients SD standard deviation 
original cohort due to clinical or radiological evidence of pathology or trauma, evidence for anatomical anomalies, etc.

\section{Pelvic incidence measurement}

The PI is defined as the angle between the line orthogonal to the inclination of the sacral endplate and the line connecting the sacral endplate with the hip axis (Fig. 1). On standard lateral radiographs there is always a deviation from the true sagittal plane leading to a certain degree of distortion of the image [22, 25]. The variability of the semiautomatic 3D PI measurement method was $0.8^{\circ}$, whereas the variability of the PI from radiographs was reported to be between $3^{\circ}$ and $6^{\circ}$ [22]. For the previously validated 3D method, the center of both femoral heads, as well as the center and inclination of the sacral endplate, was used to calculate the PI [22]. First, the femoral heads and the sacral endplate were manually selected to initialize the computerized method by defining the locations of the volumes of interest on the 3D image. Next, the computerized method automatically determined the exact centers of the femoral heads in 3D from the spheres that best fit to the 3D edges of the femoral heads. This created the true sagittal plane of the pelvis. The exact center of the sacral endplate in 3D was then automatically determined by locating the sacral endplate and finding the midpoint of the lines between the anterior and posterior
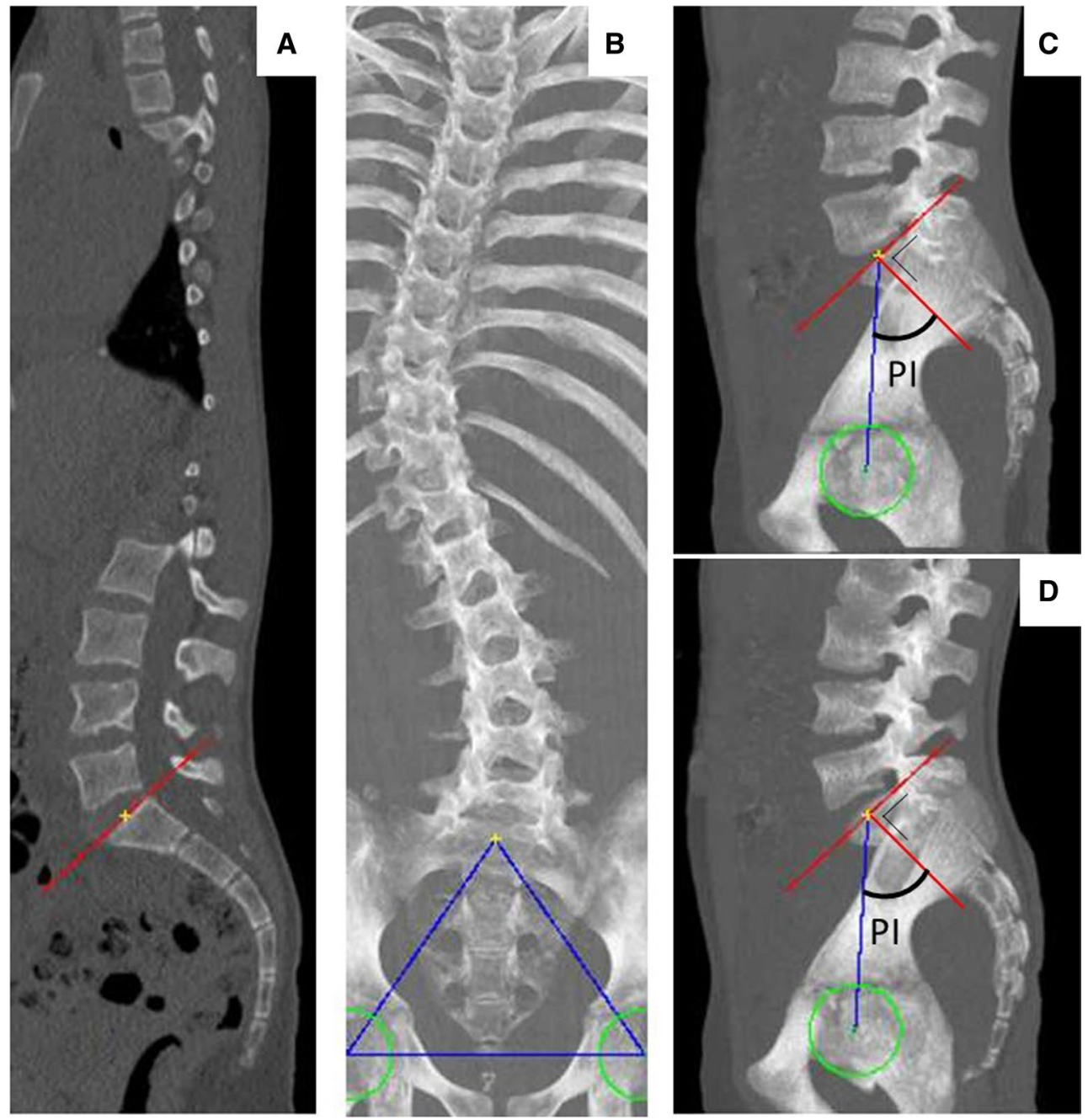

Fig. 1 Computerized measurement of the pelvic incidence (PI) on a computed tomography scan. The center and inclination of the sacral endplate $(\mathbf{a}, \mathbf{b})$ and the centers of the left and right femoral heads (c, d) were used to calculate the PI. The computerized method automatically determined the exact centers of the femoral heads in three dimensions (3D) from the spheres that best fit to the 3D edges of the femoral heads. The exact center of the sacral endplate in 3D was automatically determined by locating the sacral endplate and find- ing the midpoint of the lines between the anterior and posterior edge and between the left and right edge of the endplate. The previously validated computerized method was used to measure the PI in $3 \mathrm{D}$ images, based on previously validated image processing techniques, as the angle between the line orthogonal to the inclination of the sacral endplate and the line connecting the center of the sacral endplate with the hip axis [22] 
edge and between the left and right edge of the endplate. The previously described computerized model was used to measure the PI in 3D images, based on previously validated image processing techniques, as the angle between the line orthogonal to the inclination of the sacral endplate and the line connecting the center of the sacral endplate with the hip axis (Fig. 1) [22]. The reliability of the analysis method was previously determined, where a high consistency between manual and computerized pelvic incidence measurements (intraclass correlation coefficient: 0.961) and high interobserver reliability (0.994) was observed [22].

\section{Statistical analysis}

Statistical analyses were performed using SPSS 22.0 for Windows (SPSS Inc., Chicago, IL, USA). Descriptive statistics were computed providing means, ranges and standard deviations. The PI of the patients with AIS and controls, as well as between Lenke type 1 and 5, curves with different apex levels and curves with different lumbar modifiers, was compared using the unpaired $t$ test [26]. Pearson correlation coefficient $(r)$ defined the relationship between the PI, Cobb angle, TK and LL. The statistical significance level was set at 0.05 for all analyses.

\section{Results}

\section{Population}

CT scans of 43 preoperative AIS patients contained the whole spine as well as both femoral heads. However, six subjects had to be excluded (five male patients and one patient with a left convex thoracic curve). The final study population consisted of 37 female AIS subjects with an average age of $15.4 \pm 2.4$ years and 44 non-scoliotic age- and sex-matched controls. Demographics and curve characteristics are shown in Table 1. Within the AIS groups, the Lenke type 1 patients were on average $15.4 \pm 2.3$ years of age and the Lenke type 5 patients $15.8 \pm 3.1$ years of age $(p=0.699)$.

\section{Pelvic incidence}

The PI was on average $46.8^{\circ} \pm 12.4^{\circ}$ in AIS and $41.3^{\circ} \pm 11.4^{\circ}$ in controls ( $p=0.025$; Fig. 2$)$. The PI in the Lenke type 5 group was $50.6^{\circ} \pm 16.2^{\circ}$ and $45.9^{\circ} \pm 12.2^{\circ}$ in the Lenke type 1 group ( $p=0.384$ ). A statistically significant difference was observed between the Lenke type 5 group and the controls $(p=0.042)$, whereas the Lenke type 1 group and the controls showed no significant difference $(p=0.141 ;$ Fig. 2$)$. No significant correlations were observed between the PI and the thoracic $(r=-0.07, p=0.69)$ and lumbar Cobb angle $(r=-0.15, p=0.36)$. The PI did not differ significantly $(p \geq 0.053)$ between the curves with different lumbar modifiers; A $(n=10), \mathrm{B}(n=5)$ and $\mathrm{C}(n=6)$, according to the Lenke classification. No significant PI differences were seen between curves with different levels of the apex (thoracic: $p \geq 0.769$, lumbar: $p \geq 0.298$ ).

\section{Sagittal alignment in AIS}

Correlation analyses revealed moderate correlations between the LL (DRRs: $47.8^{\circ} \pm 9.7^{\circ}$ and radiographs: $58.2^{\circ} \pm 11.1^{\circ}$ ) and PI (DRRs: $r=0.60 ; p<0.001$ and radiographs: $r=0.64$; $p<0.001)$; however, no significant correlation was observed between the TK (DRRs: $18.9^{\circ} \pm 6.7^{\circ}$ and radiographs:
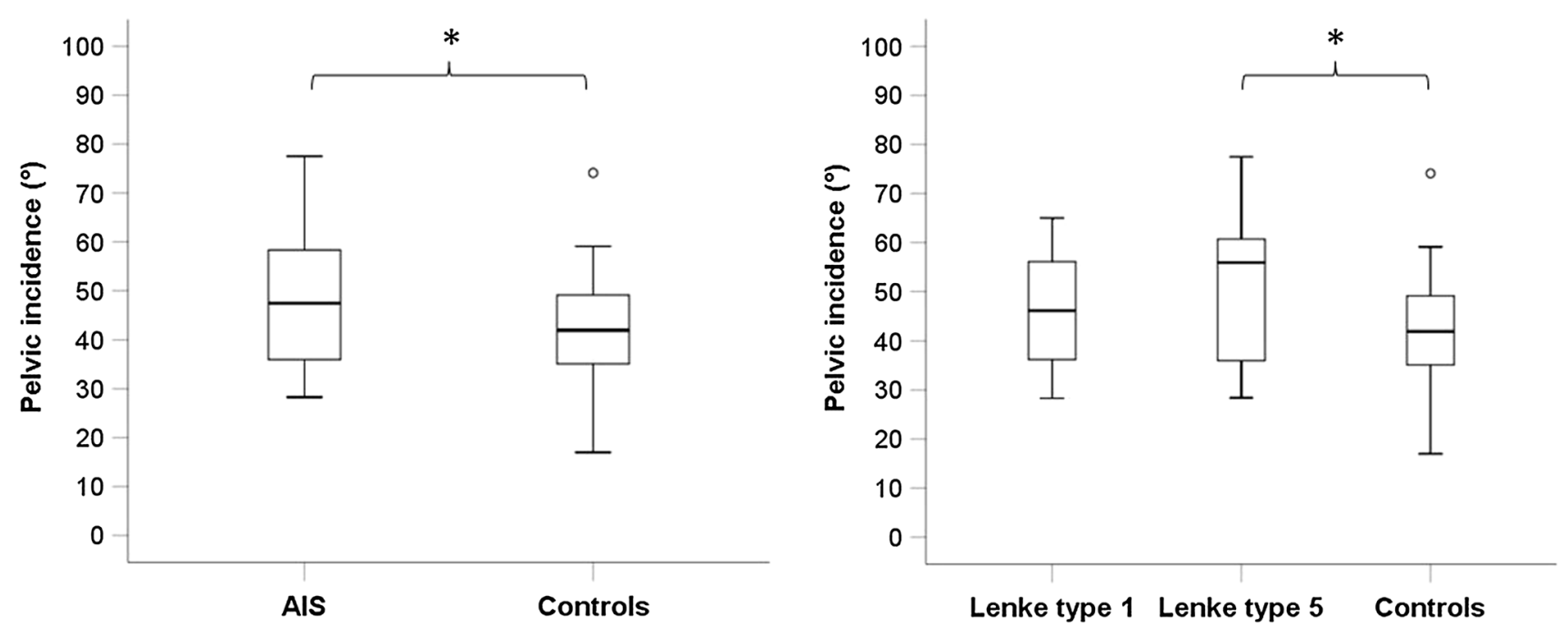

Fig. 2 Boxplots of the pelvic incidence for (left) the patients with adolescent idiopathic scoliosis (AIS) and controls, and (right) the typical primary thoracic AIS curves (Lenke type 1), typical primary (thoraco)lumbar AIS curves (Lenke type 5) and controls. *Significant difference 
$27.5^{\circ} \pm 10.6^{\circ}$ ) and PI (DRRs: $r=0.05 ; p=0.791$ and radiographs: $r=0.18 ; p=0.280)$. A moderate correlation was found between the TK and LL (DRRs: $r=0.51 ; p=0.001$ and radiographs: $r=0.58 ; p<0.001)$.

\section{Discussion}

AIS is a disease of the human spine and trunk in which fully upright sagittal spinal biomechanics is known to play an important role [1-7, 10, 27]. Since its introduction in 1985, the PI has been used to describe pelvic morphology and its relation to the spino-pelvic alignment, as well as the relationship between the spino-pelvic alignment and spinal deformities [1, 8-10]. However, previous studies used predominantly $2 \mathrm{D}$ imaging that could initiate a certain degree of distortion of the image and influences the spinopelvic measurements [25]. Therefore, the true relationship between the 3D pelvic morphology and spinal deformity has remained unclear up to now [22].

Using the accurate 3D measurement on CT scans, the PI was on average higher in patients with a Lenke type 5 curve as compared to controls, whereas the PI in patients with a Lenke type 1 curve did not differ from controls. In previous studies, the mean PI varied between $41^{\circ}$ and $49^{\circ}$ for non-scoliotic adolescents and between $42^{\circ}$ and $57^{\circ}$ for AIS patients, using radiographs $[1,10,13-15,17,19-22,28]$. In these studies, the patients with scoliosis had a moderate to severe thoracic curve (mean Cobb angles varied between $35^{\circ}$ and $60^{\circ}$ ); however, Schlösser et al. [15] included only mild curves (Cobb angle $20^{\circ}$ or lower) and found similar results (PI for thoracic AIS patients: $47^{\circ}$, PI for (thoraco)lumbar AIS patients: $42^{\circ}$ and PI for controls: $43^{\circ}$; without significant differences). It has been described that the PI in AIS is higher as compared to the non-scoliotic controls; however, others described no significant differences between AIS patients and non-scoliotic adolescents [1, 10, 13, 19, 21, 27, 28]. Slight PI differences among studies could be explained by the differences in age, since the PI increases with age, but also to the inaccuracy of the 2D method [16, 29].

The 3D PI of the non-scoliotic population was already described by Vrtovec et al. [16] and Schlösser et al. [22]. Pasha et al. [14] introduced novel pelvic parameters, based on 3D reconstructed radiographs of AIS patients and controls. However, the PI of AIS patients was only based on 2D radiographs, whereas the PI of our study was measured using low-dose CT data and a computerized method based on accurate image processing techniques [22]. Mac-Thiong et al. [10] and Farshad et al. [13] found that the scoliotic curve type was not associated with a specific pattern of sagittal pelvic morphology and balance. However, the results of the present study showed a higher PI in Lenke curve type 5 as compared to Lenke curve type 1 . By using low-dose
CT data and accurate image processing techniques, we were able to quantify the PI on 3D images with high accuracy and reproducibility in AIS patients versus controls. Using this 3D CT measurement method, bias due to image acquisition or subject positioning was avoided.

Radiographical measurements may be inaccurate due to the projective nature of radiograph acquisition, because it is usually impossible to obtain the superposition of the two femoral heads [22, 30, 31]. Moreover, the inclination of the sacral end plate in the sagittal plane may be altered by its architecture and inclination in the coronal plane. 3D reconstructed images are not affected by the characteristics of radiographic imaging and generate true sagittal views by aligning the centers of the femoral heads in $3 \mathrm{D}$, resulting in a lower variability as compared to the radiographical measurements [22]. However, if the PI is measured from 3D CT images, it is important to consider that measurements in 2D radiographic images may be overestimated by approximately $5^{\circ}[22]$. CT measurements provide superior imaging precision; however, this study does not imply that measurements of PI from plain radiographic images for clinical use should be replaced by CT.

The PI was correlated with the LL, but not with the Cobb angle or the TK. Most studies described a relation between the PI and the (thoraco)lumbar sagittal alignment, but not between the PI and the thoracic alignment $[10,13,17,19$, 21, 27, 30]. This suggests that the PI influences the (thoraco) lumbar region of the spine, but the thoracic part is independent of the PI. However, the LL is correlated with the TK, indicating that the TK is not completely independent of the PI. Additionally, we found a relation between the location of the curve, thoracic or lumbar, and the PI. The PI was higher in primary lumbar curves as compared to controls, whereas the primary thoracic curves did not differ significantly from the controls. This suggests a correlation between the PI and the pathogenesis of the scoliosis, even more because the PI is not influenced by the severity of the curve, suggesting that the PI is not influenced by spinal deformities. However, the exact role of the PI could not be determined in this cross-sectional study. In previous studies it was shown that an excess of posterior shear leads to a decrease in the rotational stiffness of the involved segments, that only posteriorly inclined vertebrae take part in the development of different scoliotic curve patterns, and that more posteriorly inclined vertebrae are rotationally less stable and could lead to initiation and progression of AIS [7, 15, 17, 32]. The fact that the PI was higher in AIS patients with primary (thoraco)lumbar curves as compared to controls, in contrary of patients with AIS with primary thoracic curves, is consistent with this theory and suggests that the PI is part of the etiopathogenesis of AIS as well as a determining factor for the curve type.

The examinations in this study were made in supine positions. Philippot et al. [33] has shown that positioning 
(standing, supine or sitting position) does not influence the PI. Because all scans were acquired preoperatively, only moderate to severe AIS curves, no mild AIS curves, were included in this study. Visualizing the femoral heads is not part of the standard low-dose (20 times lower than that of a standard CT for trauma) imaging protocol $[23,24]$. Therefore, only a small number of scans could be included for the purpose of this study. Minor, not significant, differences were observed between the Lenke type 1 group and the controls and between the Lenke type 1 group and the Lenke type 5 group. Using an alpha error probability of 0.05 , a power (1-beta error probability) of 0.80 and the means and standard deviations as described in this study, the power analysis showed a minimum sample size of 101 patients in each group to determine the differences between these groups. However, this study highlighted that the PI was higher in the Lenke type 5 curves, as compared to controls, whereas the Lenke type 1 curves did not differentiate from the normal anatomy.

\section{Conclusion}

Lenke type 5 curves show a significantly higher PI than controls, whereas the PI in Lenke type 1 curves did not differ from non-scoliotic controls. This suggests a role of pelvic morphology and spino-pelvic alignment in the pathogenesis of idiopathic scoliosis. Further longitudinal studies should explore the exact role of the PI in the initiation and progression of different types of idiopathic scoliosis.

\section{Compliance with ethical standards}

Conflict of interest We have no conflicts of interests. The research was financially supported by a research grant of K2M, Alexandre Suerman $\mathrm{Md} / \mathrm{PhD}$ grant and grants from the Swedish Society of Spinal surgeons.

Open Access This article is distributed under the terms of the Creative Commons Attribution 4.0 International License (http://creativeco mmons.org/licenses/by/4.0/), which permits unrestricted use, distribution, and reproduction in any medium, provided you give appropriate credit to the original author(s) and the source, provide a link to the Creative Commons license, and indicate if changes were made.

\section{References}

1. Legaye J, Duval-Beaupere G, Hecquet J, Marty C (1997) Pelvic incidence: a fundamental pelvic parameter for three-dimensional regulation of spinal sagittal curves. Eur Spine J 7:99-103

2. Marty C, Boisaubert B, Descamps H et al (2002) The sagittal anatomy of the sacrum among young adults, infants, and spondylolisthesis patients. Eur Spine J 11:119-125
3. Roussouly P, Nnadi C (2010) Sagittal plane deformity: an overview of interpretation and management. Eur Spine J 19:1824-1836

4. Cheng JC, Castelein RM, Chu WC et al (2015) Adolescent idiopathic scoliosis. Nat Rev Disease Primers 1:15030

5. Dickson RA, Lawton JO, Archer IA, Butt WP (1984) The pathogenesis of idiopathic scoliosis. Biplanar spinal asymmetry. J Bone Jt Surg Br 66:8-15

6. Schlösser TP, Janssen MM, Hogervorst T et al (2017) The odyssey of sagittal pelvic morphology during human evolution: a perspective on different hominoidae. Spine J 17(8):1202-1206

7. Castelein RM, van Dieen JH, Smit TH (2005) The role of dorsal shear forces in the pathogenesis of adolescent idiopathic scoliosis-a hypothesis. Med Hypotheses 65:501-508

8. During J, Goudfrooij H, Keessen W, Beeker TW, Crowe A (1985) Toward standards for posture. Postural characteristics of the lower back system in normal and pathologic conditions. Spine 10:83-87

9. Duval-Beaupere G, Schmidt C, Cosson P (1992) A barycentremetric study of the sagittal shape of spine and pelvis: the conditions required for an economic standing position. Ann Biomed Eng 20:451-462

10. Mac-Thiong JM, Labelle H, Charlebois M, Huot MP, de Guise JA (2003) Sagittal plane analysis of the spine and pelvis in adolescent idiopathic scoliosis according to the coronal curve type. Spine 28:1404-1409

11. Roussouly P, Pinheiro-Franco JL (2011) Biomechanical analysis of the spino-pelvic organization and adaptation in pathology. Eur Spine J 20(Suppl 5):609-618

12. Le Huec JC, Aunoble S, Philippe L, Nicolas P (2011) Pelvic parameters: origin and significance. Eur Spine J 20(Suppl 5):564-571

13. Farshad M, Catanzaro S, Schmid SL (2016) The spinopelvic geometry in different Lenke curve types of adolescent idiopathic scoliosis. Spine Deform 4:425-431

14. Pasha S, Aubin CE, Sangole AP, Labelle H, Parent S, MacThiong JM (2014) Three-dimensional spinopelvic relative alignment in adolescent idiopathic scoliosis. Spine 39:564-570

15. Schlösser TP, Shah SA, Reichard SJ, Rogers K, Vincken KL, Castelein RM (2014) Differences in early sagittal plane alignment between thoracic and lumbar adolescent idiopathic scoliosis. Spine J 14:282-290

16. Schlösser TP, Janssen MM, Vrtovec T et al (2014) Evolution of the ischio-iliac lordosis during natural growth and its relation with the pelvic incidence. Eur Spine J 23:1433-1441

17. Schlösser TP, Vincken KL, Rogers K, Castelein RM, Shah SA (2015) Natural sagittal spino-pelvic alignment in boys and girls before, at and after the adolescent growth spurt. Eur Spine J 24:1158-1167

18. Borges PA, Ocampos GP, Mancuso Filho JA, Letaif OB, Marcon RM, Cristante AF (2014) The sagital balance in idiopatic and neuromuscular scoliosis. Acta Ortop Bras 22:179-182

19. Clement JL, Geoffray A, Yagoubi F et al (2013) Relationship between thoracic hypokyphosis, lumbar lordosis and sagittal pelvic parameters in adolescent idiopathic scoliosis. Eur Spine J 22:2414-2420

20. La Maida GA, Zottarelli L, Mineo GV, Misaggi B (2013) Sagittal balance in adolescent idiopathic scoliosis: radiographic study of spino-pelvic compensation after surgery. Eur Spine J 22(Suppl 6):S859-S867

21. Yong Q, Zhen L, Zezhang Z et al (2012) Comparison of sagittal spinopelvic alignment in chinese adolescents with and without idiopathic thoracic scoliosis. Spine 37:E714-E720

22. Vrtovec T, Janssen MM, Pernus F, Castelein RM, Viergever MA (2012) Analysis of pelvic incidence from 3-dimensional images of a normal population. Spine 37:E479-E485 
23. Kalra MK, Quick P, Singh S, Sandborg M, Persson A (2013) Whole spine CT for evaluation of scoliosis in children: feasibility of sub-milli-Sievert scanning protocol. Acta Radiol $54: 226-230$

24. Abul-Kasim K, Overgaard A, Maly P, Ohlin A, Gunnarsson M, Sundgren PC (2009) Low-dose helical computed tomography (CT) in the perioperative workup of adolescent idiopathic scoliosis. Eur Radiol 19:610-618

25. Ghostine B, Sauret C, Assi A et al (2017) Influence of patient axial malpositioning on the trueness and precision of pelvic parameters obtained from $3 \mathrm{D}$ reconstructions based on biplanar radiographs. Eur Radiol 27:1295-1302

26. Lenke LG, Edwards CC, Bridwell KH (2003) The Lenke classification of adolescent idiopathic scoliosis: how it organizes curve patterns as a template to perform selective fusions of the spine. Spine 28:S199-S207

27. Upasani VV, Tis J, Bastrom T et al (2007) Analysis of sagittal alignment in thoracic and thoracolumbar curves in adolescent idiopathic scoliosis: how do these two curve types differ? Spine 32:1355-1359

28. Roussouly P, Labelle H, Rouissi J, Bodin A (2013) Pre- and postoperative sagittal balance in idiopathic scoliosis: a comparison over the ages of two cohorts of 132 adolescents and 52 adults. Eur Spine J 22(Suppl 2):S203-S215

29. Vrtovec T, Janssen MM, Likar B, Castelein RM, Viergever MA, Pernus F (2013) Evaluation of pelvic morphology in the sagittal plane. Spine J 13:1500-1509

30. Vaz G, Roussouly P, Berthonnaud E, Dimnet J (2002) Sagittal morphology and equilibrium of pelvis and spine. Eur Spine $\mathbf{J}$ 11:80-87

31. Glaser DA, Doan J, Newton PO (2012) Comparison of 3-dimensional spinal reconstruction accuracy: biplanar radiographs with EOS versus computed tomography. Spine 37:1391-1397

32. Kouwenhoven JW, Smit TH, van der Veen AJ, Kingma I, van Dieen JH, Castelein RM (2007) Effects of dorsal versus ventral shear loads on the rotational stability of the thoracic spine: a biomechanical porcine and human cadaveric study. Spine 32:2545-2550

33. Philippot R, Wegrzyn J, Farizon F, Fessy MH (2009) Pelvic balance in sagittal and lewinnek reference planes in the standing, supine and sitting positions. Orthop Traumatol Surg Res 95:70-76

\section{Affiliations}

\section{Rob C. Brink ${ }^{1}$ (D) . Ludvig Vavruch ${ }^{2,3} \cdot$ Tom P. C. Schlösser $^{1} \cdot$ Kasim Abul-Kasim $^{4} \cdot$ Acke Ohlin $^{5} \cdot$ Hans Tropp $^{2,3}$. René M. Castelein ${ }^{1} \cdot$ Tomaž Vrtovec $^{6}$}

René M. Castelein

r.m.castelein@umcutrecht.nl

1 Department of Orthopaedic Surgery, G05.228, University Medical Center Utrecht, P.O. Box 85500, 3508 GA Utrecht, The Netherlands

2 Department of Clinical and Experimental Medicine, Linköping University, Linköping, Sweden

3 Center for Medical Image Science and Visualization, Linköping University, Linköping, Sweden
4 Division of Neuroradiology, Diagnostic Centre for Imaging and Functional Medicine, Faculty of Medicine, Lund University, Skåne University Hospital, Malmö, Sweden

5 Department of Orthopaedic Surgery, Faculty of Medicine, Lund University, Skåne University Hospital, Malmö, Sweden

6 Faculty of Electrical Engineering, University of Ljubljana, Ljubljana, Slovenia 